\title{
Experimental Verification of Wide-Field-Integration of Optic Flow for State Estimation
}

\author{
By Naoto KoBAYASHI, ${ }^{1)}$ Masataka OISHI, ${ }^{1)}$ Yutaka KINJO ${ }^{1)}$ and Shinji HoKAмOTO ${ }^{1)}$ \\ ${ }^{1)}$ Department of Aeronautics and Astronautics, Kyushu University, Fukuoka, Japan
}

(Received July 31st, 2015)

\begin{abstract}
This paper discusses the performance of Wide-Field-Integration (WFI) of optic flow when it is applied to a guidance and navigation system for space probes near asteroid surface. WFI of optic flow is a state estimation method inspired by the visual processing system of compound eyes of flying insects. This method has attractive features: low computational cost, applicableness to a lowresolution image sensor, and robustness for unknown environments. Unlike the theory of WFI of optic flow, the region of an image sensor is limited in a real system. In this paper, the effects of the directions of optical axes on estimation accuracy are investigated in numerical simulations and experiments. The evaluation results in simulations indicate the proper directions of optic flow sensors. The results also indicate that under a limited field of view, one optic flow sensor has frequently difficulty to distinguish motion variables from WFI of optic flow. Finally, the results obtained in numerical simulations are examined in experiments by using two image sensors.
\end{abstract}

Key Words: Optic Flow, Wide-Field-Integration, Experimental System, Image Region

\begin{tabular}{cll} 
Nomenclature & & \\
$\boldsymbol{d}$ & $:$ & distance vector to a fiducial point \\
$\boldsymbol{e}$ & $:$ & unit vector on a tangent plane \\
$\boldsymbol{F}$ & $:$ & vector-valued sensitivity function \\
$j$ & $:$ & index of sensitivity functions \\
$k$ & $:$ & index of sensor points \\
$M$ & $:$ & total number of sensitivity functions \\
$N$ & $:$ & total number of sensor points \\
$p$ & $:$ & roll rate \\
$q$ & $:$ & pitch rate \\
$\boldsymbol{Q}$ & $:$ & line-of-sight vector \\
$\dot{\boldsymbol{Q}}$ & $:$ & vector-valued optic flow \\
$r$ & $:$ & yaw rate \\
$u$ & $:$ & forward velocity \\
$v$ & $:$ & lateral velocity \\
$\boldsymbol{v}$ & $:$ & translational velocity vector \\
$w$ & $:$ & vertical velocity \\
$X$ & $:$ & forward position in camera frame \\
$\boldsymbol{X}$ & $:$ & state vector \\
$x$ & $:$ & forward position in world frame \\
$\boldsymbol{x}$ & $:$ & velocity dependent state vector \\
$Y$ & $:$ & lateral position in camera frame \\
$y$ & $:$ & lateral position in world frame \\
$\boldsymbol{y}$ & $:$ & wide-field-integration measurement vector \\
$z$ & $:$ & vertical position in world frame \\
$\beta$ & $:$ & elevation angle \\
$\gamma$ & $:$ & azimuth angle \\
$\theta$ & $:$ & pitch angle \\
$\mu$ & $:$ & nearness function \\
$\phi$ & $:$ & roll angle \\
$\psi$ & $:$ & yaw angle \\
$\Omega$ & $:$ & solid angle on spherical image plane \\
$\boldsymbol{\omega}$ & $:$ & angular velocity vector \\
Subscripts & & \\
& & \\
& & \\
\hline &
\end{tabular}

$\begin{array}{cll}b & : & \text { expression in body reference frame } \\ c & : & \text { expression in camera frame } \\ \text { pho } & : & \text { photoreceptor } \\ s & : & \text { expression in spherical frame } \\ t & : & \text { expression in terrain reference frame }\end{array}$

\section{Introduction}

Autonomy is a key technology for space exploration missions. Remote control from Earth is effective for near space missions like activities on Moon. However, deep space exploration missions, like activities on asteroids, need autonomous technologies because of extremely long delay time of signals. Thus an autonomous system is indispensable especially for activity near asteroid surface, because quick response is required. ${ }^{1,2)}$

This study discusses how to apply Wide-Field-Integration (WFI) of optic flow to a guidance and navigation system for space probes near asteroid surface. Optic flow is the vector field of relative velocities produced by motion of a projected image over the surface of the retina. ${ }^{3,4)}$ Some flying insects, e.g., bees, flies, and dragonflies, are said to utilize optic flow for their motion control and collision avoidance. ${ }^{5)}$ WFI of optic flow is a state estimation method inspired by the visual processing system of flying insects' compound eyes. ${ }^{6-11)}$ This method has attractive features: low computational cost, applicableness to a low-resolution image sensor, and robustness for unknown environments. These simple, robust, and lightweight characteristics are useful for autonomous systems, especially under the constraints on power, weight, and size. Thus WFI of optic flow is applied to estimate states of space probes in this research.

However, WFI of optic flow is hardly considered in guidance and navigation system of space probes because of its difficulties in application to real systems. The most critical one is that optic flow is assumed in its theory to be obtained on a whole spherical image surface, although an image region of a real system 
is limited and flat. Furthermore, in a real system, any sensor signals are inevitably contaminated by noises. However, the effect of sensor noises of real systems has not been discussed yet. Thus, the estimation accuracy for state parameters must be investigated experimentally.

In this paper, the effects of the directions of optical axes on estimation accuracy are investigated by numerical simulations and experiments. This investigation contributes to arrange the mounting angles of image sensors in applying WFI of optic flow to a real system. In the numerical simulations, the estimation accuracies are compared in the mean values and standard deviations of the estimation error, for the different azimuth and elevation directions of optical axes. Subsequently in the experiments, estimation accuracy of optic flow sensors is examined by using real systems mounted on a multirotor vehicle, which is an experimental model of space probes moving near asteroid surface. Moreover, the estimated motion variables are compared with the outputs of a motion capture system, and the effects of the directions of optical axes are discussed.

\section{Description of Wide-Field-Integration Method}

\subsection{Optic flow model}

Figure 1 shows a space probe flying near the surface of a planet. Spherical image surface is set around the center of the gravity of the vehicle, and optic flow data is obtained from each photoreceptor on it. Line-of-sight vector is written with elevation $\beta$ and azimuth $\gamma$ angles as follows:

$$
\boldsymbol{Q}=\left[\begin{array}{lll}
\sin \beta \cos \gamma & \sin \beta \sin \gamma & \cos \beta
\end{array}\right]^{T}
$$

The fiducial point on the surface is assumed stationary relative to the reference frame fixed on the terrain. The motion parallax $\dot{\boldsymbol{Q}}$ is the time derivative of $\boldsymbol{Q}$ on the spherical image surface, which is caused by the motion of translational and angular velocities of the probe. Let $v=[u v w]^{T}$ be the translational velocity and $\omega=\left[\begin{array}{lll}p & q\end{array}\right]^{T}$ be the angular velocity. Then optic flow is ${ }^{6)}$

$$
\dot{\boldsymbol{Q}}=-\omega \times \boldsymbol{Q}-\mu(\boldsymbol{v}-\langle\boldsymbol{v}, \boldsymbol{Q}\rangle \boldsymbol{Q})
$$

where $\langle *, *\rangle$ denotes the inner product, and the nearness function $\mu=1 /\left|d_{p h o}\right|$ is the inverse of the distance to a point on the terrain. The optic flow can be divided to $\gamma$ and $\beta$ components like $\dot{\boldsymbol{Q}}=\dot{Q}^{\gamma} \boldsymbol{e}_{\gamma}+\dot{Q}^{\beta} \boldsymbol{e}_{\beta}$, and they are written as follows:

$$
\begin{aligned}
& \dot{Q}^{\gamma}=p c \beta c \gamma+q c \beta s \gamma-r s \beta+\mu(u s \gamma-v c \gamma) \\
& \dot{Q}^{\beta}=p s \gamma-q c \gamma-\mu(u c \beta c \gamma+v c \beta s \gamma-w s \beta)
\end{aligned}
$$

where $c(*)$ and $s(*)$ mean $\cos (*)$ and $\sin (*)$, respectively. If the surrounding environment is modeled as a flat, infinite, and horizontal plane, the nearness function $\mu$ can be written as

$$
\mu=\frac{-s \beta c \gamma s \theta+s \beta s \gamma s \phi c \theta+c \beta c \phi c \theta}{z}
$$

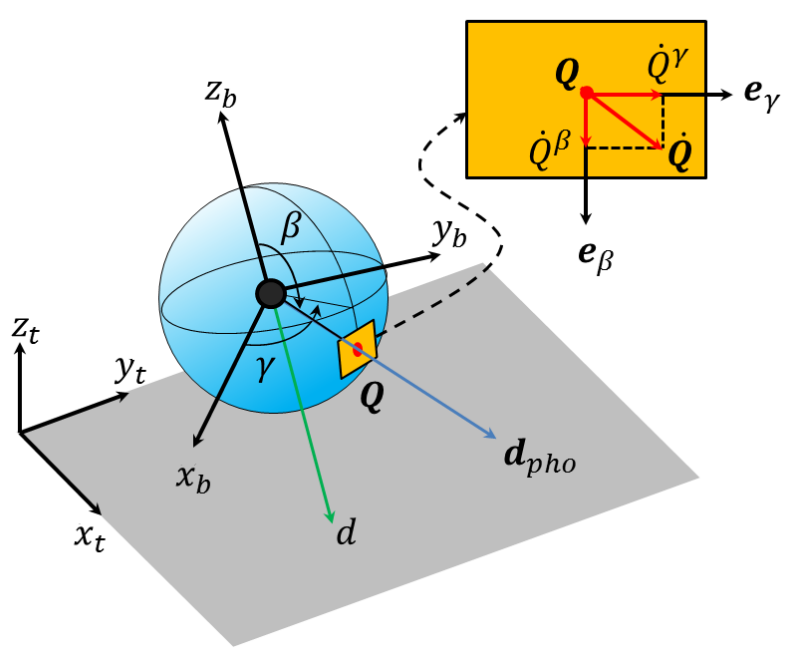

Fig. 1. Model of Wide-Field-Integration of optic flow.

\subsection{Wide-Field-Integration}

When a sensitivity function is expressed as $\boldsymbol{F}_{\boldsymbol{j}}=F_{j}^{\gamma} \boldsymbol{e}_{\gamma}+$ $F_{j}^{\beta} \boldsymbol{e}_{\beta}$, the $j$ th WFI output is defined as

$$
y_{j}=\int \dot{\boldsymbol{Q}} \cdot \boldsymbol{F}_{\boldsymbol{j}} d \Omega=\int\left(\dot{Q}^{\gamma} F_{j}^{\gamma}+\dot{Q}^{\beta} F_{j}^{\beta}\right) d \Omega
$$

where $d \Omega=\sin \beta d \beta d \gamma$ is a solid angle on the imaging surface sphere. This paper uses spherical harmonics as a sensitivity function. For the $1 \mathrm{st}-M$ th sensitivity functions, one can construct an $M \times 1$ measurement vector of WFI outputs as

$$
\begin{aligned}
& \boldsymbol{y}=\left[\begin{array}{lll}
y_{1} & \cdots & y_{M}
\end{array}\right]^{T} \\
& =\left[\begin{array}{lll}
\int\left(\begin{array}{lll}
\left.\dot{Q}^{\gamma} F_{1}^{\gamma}+\dot{Q}^{\beta} F_{1}^{\beta}\right) d \Omega & \cdots & \int\left(\dot{Q}^{\gamma} F_{M}^{\gamma}+\dot{Q}^{\beta} F_{M}^{\beta}\right.
\end{array}\right) d \Omega & ]^{T}
\end{array}\right.
\end{aligned}
$$

Generally, Eq. (7) is used as the equation of WFI output. However, to distinguish the effects on two directions, the following equations are adopted in this paper as the equations of WFI output.

$$
\begin{gathered}
\boldsymbol{y}=\left[\begin{array}{ll}
\left(\boldsymbol{y}^{\gamma}\right)^{T} & \left(\boldsymbol{y}^{\beta}\right)^{T}
\end{array}\right]^{T} \\
\boldsymbol{y}^{\gamma}=\left[\begin{array}{lll}
\int \dot{Q}^{\gamma} F_{1}^{\gamma} d \Omega & \cdots & \int \dot{Q}^{\gamma} F_{M}^{\gamma} d \Omega
\end{array}\right]^{T} \\
\boldsymbol{y}^{\beta}=\left[\begin{array}{lll}
\int \dot{Q}^{\beta} F_{1}^{\beta} d \Omega & \cdots & \int \dot{Q}^{\beta} F_{M}^{\beta} d \Omega
\end{array}\right]^{T}
\end{gathered}
$$

where WFI output $\boldsymbol{y}=\left[\begin{array}{lllll}y_{1}^{\gamma} & \cdots & y_{M}^{\gamma} y_{1}^{\beta} \cdots & y_{M}^{\beta}\end{array}\right]^{T}$ is a $2 M \times 1$ measurement vector.

\subsection{State estimation}

In a standard theory, optic flow $\dot{\boldsymbol{Q}}$ is regarded as a continuous function, and WFI output is calculated from the integration of the inner product between $\dot{\boldsymbol{Q}}$ and $\boldsymbol{F}$. However, in a real system, the optic flow data is obtained at discretized points on an image sensor. Thus, approximating the integration by Riemann sums, 
we obtain the actual WFI output as follows:

$$
\boldsymbol{y}=\left[\begin{array}{c}
\sum_{k=1}^{N} \dot{Q}^{\gamma}\left(\gamma_{k}, \beta_{k}, \boldsymbol{x}\right) F_{1}^{\gamma}\left(\gamma_{k}, \beta_{k}\right) \Delta \Omega \\
\vdots \\
\sum_{k=1}^{N} \dot{Q}^{\gamma}\left(\gamma_{k}, \beta_{k}, \boldsymbol{x}\right) F_{M}^{\gamma}\left(\gamma_{k}, \beta_{k}\right) \Delta \Omega \\
\sum_{k=1}^{N} \dot{Q}^{\beta}\left(\gamma_{k}, \beta_{k}, \boldsymbol{x}\right) F_{1}^{\beta}\left(\gamma_{k}, \beta_{k}\right) \Delta \Omega \\
\vdots \\
\sum_{k=1}^{N} \dot{Q}^{\beta}\left(\gamma_{k}, \beta_{k}, \boldsymbol{x}\right) F_{M}^{\beta}\left(\gamma_{k}, \beta_{k}\right) \Delta \Omega
\end{array}\right]
$$

where $N$ is the number of photoreceptors on the image surface. This output $\boldsymbol{y}$ is a nonlinear function of a state vector $\boldsymbol{X}$ consisting of altitude, attitude angles, and translational/angular velocities, and it can be expressed as $\boldsymbol{y}=g(\boldsymbol{X}) .{ }^{12)}$ However, nonlinear expressions make estimation process much complicate and time consuming. Thus, in a standard WFI of optic flow method, the nonlinear function is linearized for translational/angular velocities. Furthermore, it is assumed that attitude angles and altitude are measured with attitude sensors and a laser sensor respectively. In this paper, to focus on the effect of image sensor directions, we take the same assumption for simplicity, and estimate only the translational and angular velocities from WFI of optic flow. Note that even under the assumption of using attitude sensor, angular rate estimation from WFI of optic flow is meaningful because the estimation from differentiating attitude signals usually contains large differential error. (Let us add that a simple estimation system with fewer sensors is desirable in future, and we are continuing to research for such systems). ${ }^{12)}$

After linearization, $\boldsymbol{y}$ can be expressed for the state vector

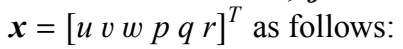

$$
\boldsymbol{y}=\boldsymbol{C}(\phi, \theta, z) \boldsymbol{x}
$$

where $\boldsymbol{C}$ is an observation matrix of $2 M \times 6$ including $(\phi, \theta, z)$ parameters. Then, the state variables can be estimated through the pseudo inverse matrix of $\boldsymbol{C}$ as follows:

$$
\boldsymbol{x}=\boldsymbol{C}^{\dagger}(\phi, \theta, z) \boldsymbol{y}
$$

\subsection{Image surface transformation}

Although optic flow is assumed in the theory to be obtained on a whole spherical image surface, the image surface of a real system is flat. Thus optic flow data obtained from a real image sensor should be transformed to the data on a spherical image surface. Figure 2 shows the geometric relation between two image surfaces. $\dot{Q}_{c}^{X}$ and $\dot{Q}_{c}^{Y}$ indicate the horizontal $\left(X_{c}\right)$ and vertical $\left(Y_{c}\right)$ components of the optic flow projected on a flat image surface. The $\dot{Q}_{c}^{X}$ and $\dot{Q}_{c}^{Y}$ are transformed to $\dot{Q}^{\gamma}$ and $\dot{Q}^{\beta}$ through the following process. For the camera mounted at $\left(\psi_{c}, \theta_{c}, \phi_{c}\right)$, the rotating matrix from the body reference frame to the camera frame $\boldsymbol{R}_{\boldsymbol{c} b}$ becomes

$$
\boldsymbol{R}_{\boldsymbol{c} \boldsymbol{b}}=\left[\begin{array}{ccc}
c \theta_{c} c \psi_{c} & c \theta_{c} s \psi_{c} & -s \theta_{c} \\
s \phi_{c} s \theta_{c} c \psi_{c}-c \phi_{c} s \psi_{c} & s \phi_{c} s \theta_{c} s \psi_{c}+c \phi_{c} c \psi_{c} & s \phi_{c} c \theta_{c} \\
c \phi_{c} s \theta_{c} c \psi_{c}+s \phi_{c} s \psi_{c} & c \phi_{c} s \theta_{c} s \psi_{c}-s \phi_{c} c \psi_{c} & c \phi_{c} c \theta_{c}
\end{array}\right]
$$

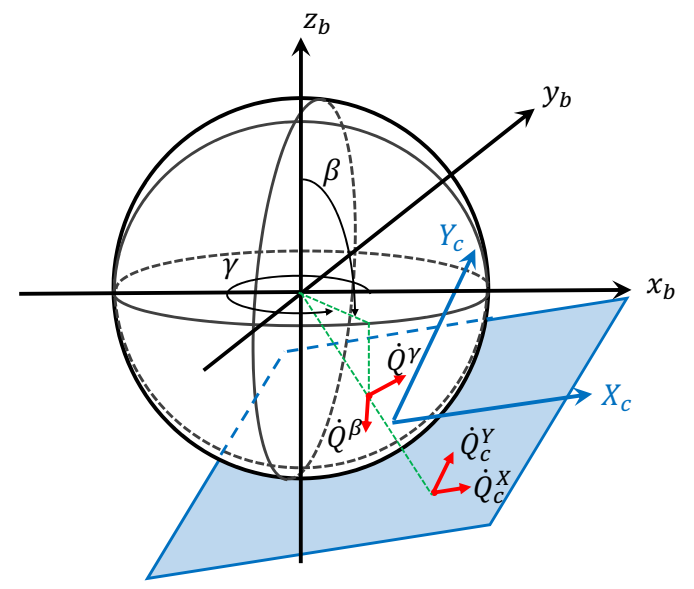

Fig. 2. Model of a flat image surface and a spherical image surface.

Similarly, the transformation matrix from the body reference frame to the spherical frame $\boldsymbol{R}_{\boldsymbol{s} b}$ is

$$
\boldsymbol{R}_{s b}=\left[\begin{array}{ccc}
s \beta c \gamma & s \beta s \gamma & c \beta \\
c \beta c \gamma & c \beta s \gamma & -s \beta \\
-s \gamma & c \gamma & 0
\end{array}\right]
$$

Thus the entire transformation from a flat surface to a spherical surface is

$$
\left[\begin{array}{c}
0 \\
\dot{Q}^{\beta} \\
\dot{Q}^{\gamma}
\end{array}\right]=\boldsymbol{R}_{\boldsymbol{s} \boldsymbol{b}} \boldsymbol{R}_{\boldsymbol{c} \boldsymbol{b}}^{-1}\left[\begin{array}{c}
\dot{Q}_{c}^{X} \\
\dot{Q}_{c}^{Y} \\
0
\end{array}\right]
$$

Note that the optic flow of the orthogonal direction on image surfaces are zero.

\section{Numerical Simulations}

As described above, optic flow in the theory is assumed to be obtained in the whole area of a spherical image surface around the vehicle's mass center. While in a real system, optic flow is obtained from an image sensor with a limited field of view. This section examines the effect of the limited field of view and the direction of image sensors on the estimation accuracy in numerical simulations, considering sensor noises.

Table 1 shows a flight condition of a space probe used in simulations: a level flight for reconnaissance of a terrain surface. From referring to a real optic flow sensor, the field of view of a camera is specified as 30 and 40 degrees in its elevation and azimuth directions, respectively. Table 2 summarizes the image

Table 1. Flight condition of a space probe used in simulations.

\begin{tabular}{|c|c|}
\hline$(\phi, \theta, \psi)[\mathrm{deg}]$ & $(0,0,0)$ \\
\hline$z[\mathrm{~m}]$ & 10 \\
\hline$(u, v, w)[\mathrm{m} / \mathrm{s}]$ & $(2.0,2.0,0)$ \\
\hline$(p, q, r)[\mathrm{deg} / \mathrm{s}]$ & $(0,0,0)$ \\
\hline
\end{tabular}

Table 2. Image region of a camera.

\begin{tabular}{|c|c|c|c|}
\hline \multicolumn{2}{|c|}{} & $\beta[\mathrm{deg}]$ & $\gamma[\mathrm{deg}]$ \\
\hline \multirow{4}{*}{ Case 1 } & $\mathrm{a}$ & $120 \sim 150$ & $-20 \sim 20$ \\
\cline { 2 - 4 } & $\mathrm{b}$ & $120 \sim 150$ & $25 \sim 65$ \\
\cline { 2 - 4 } & $\mathrm{c}$ & $120 \sim 150$ & $70 \sim 110$ \\
\hline \multirow{4}{*}{ Case 2 } & $\mathrm{a}$ & $135 \sim 165$ & $-20 \sim 20$ \\
\cline { 2 - 4 } & $\mathrm{b}$ & $120 \sim 150$ & $-20 \sim 20$ \\
\cline { 2 - 4 } & $\mathrm{c}$ & $105 \sim 135$ & $-20 \sim 20$ \\
\hline
\end{tabular}



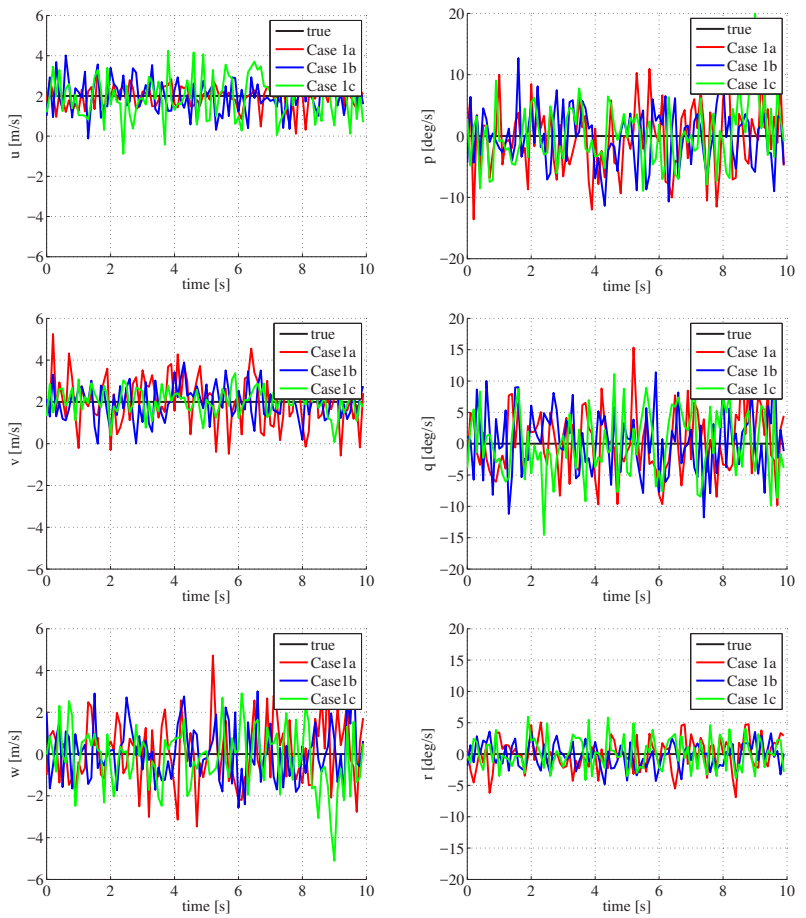

Fig. 3. Time histories of the estimation results in Case 1.
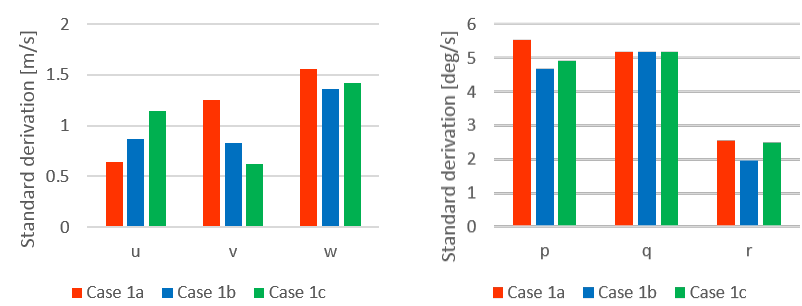

Fig. 4. Standard deviations of estimation error in Case 1.

region of a camera. The effect of the azimuth direction is investigated in Case 1, while Case 2 examines that of the elevation direction. Sensor noises included in the optic flow is assumed to be white noises, whose mean value is zero and standard deviation is $0.3 \mathrm{rad} / \mathrm{s}$. The translational and rotational velocities of a space probe are estimated from the optic flow obtained in the specified regions, and the results are compared in the mean values and standard deviations of the estimation errors.

Figure 3 shows the time histories of the state variables in Case 1. The black solid line indicates the true value, and the velocities estimated in every 0.1 second are plotted in different colors for three cases. Table 3 shows the mean values of estimation errors evaluated as a root mean square, and Fig. 4 is the standard deviations of the estimation errors. These results indicate that the estimation accuracy of $u$ becomes worse and that of $v$ becomes better according that the direction of the optical axis changes in the azimuth direction from 0 to 90 degrees, i.e. from Case 1a to Case 1c. Note that Case 1a has an image sensor facing along the $\mathrm{x}$-axis while Case $1 \mathrm{c}$ has one

\begin{tabular}{|c|c|c|c|c|c|c|}
\hline & $\begin{array}{c}u \\
{[\mathrm{~m} / \mathrm{s}]}\end{array}$ & $\begin{array}{c}v \\
{[\mathrm{~m} / \mathrm{s}]}\end{array}$ & $\begin{array}{c}w \\
{[\mathrm{~m} / \mathrm{s}]}\end{array}$ & $\begin{array}{c}p \\
{[\mathrm{deg} / \mathrm{s}]}\end{array}$ & $\begin{array}{c}q \\
{[\mathrm{deg} / \mathrm{s}]}\end{array}$ & $\begin{array}{c}r \\
{[\mathrm{deg} / \mathrm{s}]}\end{array}$ \\
\hline Case 1a & 0.48 & 0.99 & 1.25 & 4.48 & 4.21 & 2.04 \\
\hline Case $1 b$ & 0.70 & 0.69 & 1.07 & 3.79 & 4.14 & 1.61 \\
\hline Case 1c & 0.94 & 0.48 & 1.09 & 3.84 & 4.24 & 2.06 \\
\hline
\end{tabular}
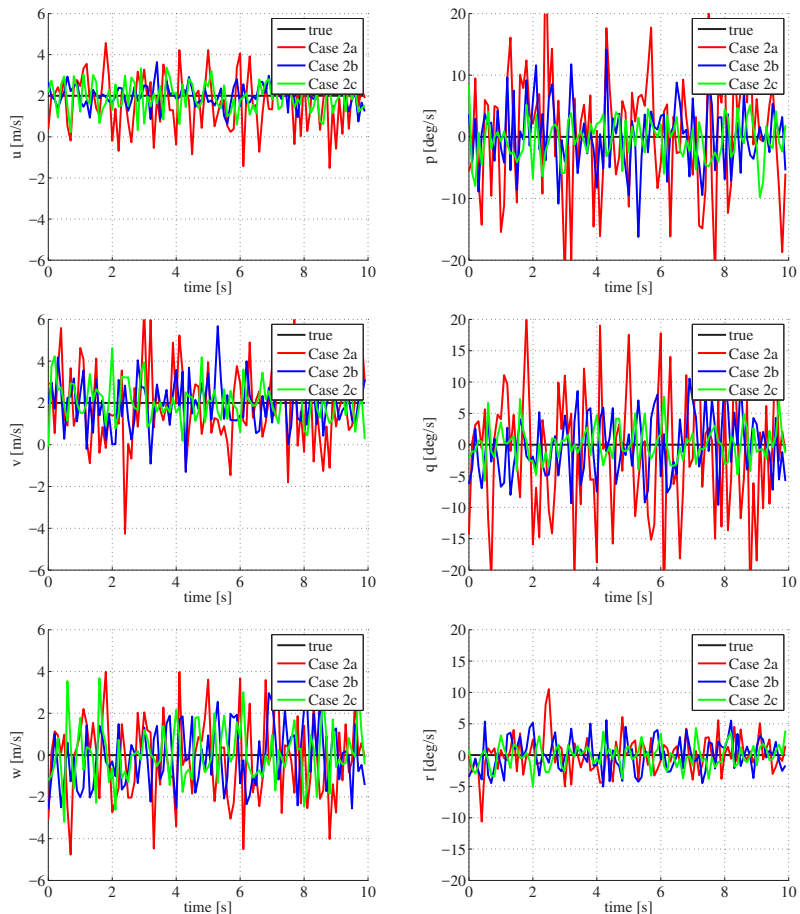

Fig. 5. Time histories of the estimation results in Case 2.
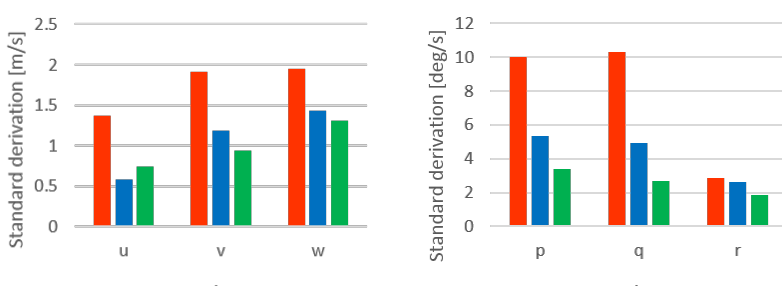

Fig. 6. Standard deviations of estimation error in Case 2.

along the y-axis. Thus, the result implies that the translational velocity estimation becomes more accurate for the direction of image sensors. Optic flow patterns obtained for a motion along its optical direction is distinctive from those for others and thus the estimation of translational velocity along an image sensor becomes better. These tendencies are kept for opposite directions of the azimuth angle, from 180 to 90 degrees, due to the symmetry of the spherical image surface. For other state parameters, there appears no dependency on the direction of the azimuth angle.

Figure 5 shows the time histories of the estimation results in Case 2. Table 4 and Fig. 6 show the mean values and the standard deviations of the estimation errors. These results indicate that the estimation accuracy of almost all state parameters become better according to the elevation direction of the optical axis changes from 150 to 120 degrees. This implies that image sensors facing nearly a horizontal direction show better accuracy than that facing a downward direction. This is because flow patterns in near horizontal regions are distinctive for dif-

Table 4. Mean values of estimation error in Case 2.
\begin{tabular}{|c|c|c|c|c|c|c|}
\hline & $\begin{array}{c}u \\
{[\mathrm{~m} / \mathrm{s}]}\end{array}$ & $\begin{array}{c}v \\
{[\mathrm{~m} / \mathrm{s}]}\end{array}$ & $\begin{array}{c}w \\
{[\mathrm{~m} / \mathrm{s}]}\end{array}$ & $\begin{array}{c}p \\
{[\mathrm{deg} / \mathrm{s}]}\end{array}$ & $\begin{array}{c}q \\
{[\mathrm{deg} / \mathrm{s}]}\end{array}$ & $\begin{array}{c}r \\
{[\mathrm{deg} / \mathrm{s}]}\end{array}$ \\
\hline Case $2 \mathrm{a}$ & 1.08 & 1.52 & 1.53 & 8.04 & 8.15 & 2.11 \\
\hline Case $2 \mathrm{~b}$ & 0.45 & 0.94 & 1.21 & 4.18 & 4.11 & 2.11 \\
\hline Case $2 \mathrm{c}$ & 0.62 & 0.74 & 0.99 & 2.75 & 2.05 & 1.44 \\
\hline
\end{tabular}




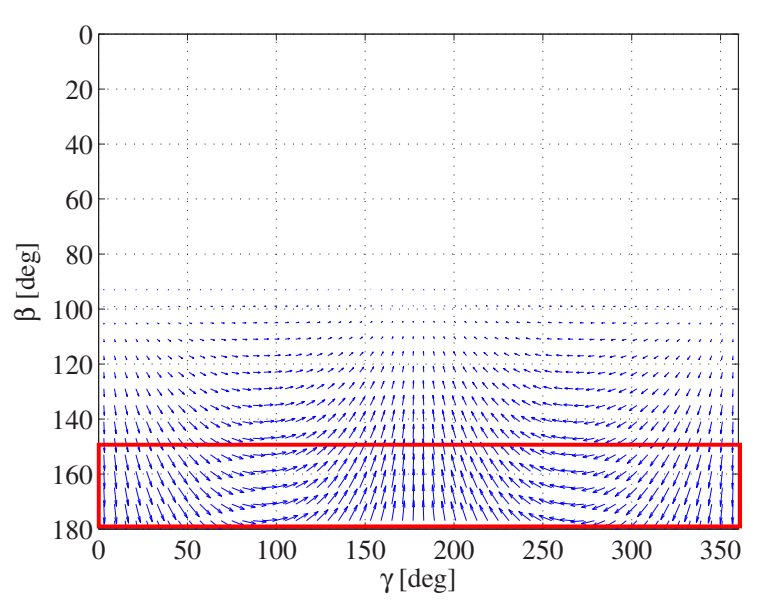

Fig. 7. Example of optic flow produced by $u$ motion.

Table 5. Specifications of the optic flow sensor.

\begin{tabular}{|c|c|}
\hline Resolution $(\mathrm{H}) \times(\mathrm{V})[$ pixel] & $320 \times 240$ \\
\hline Number of optic flow data $(\mathrm{H}) \times(\mathrm{V})$ & $79 \times 59$ \\
\hline Field of view $(\mathrm{H}) \times(\mathrm{V})[\mathrm{deg}]$ & $45.1 \times 34.6$ \\
\hline Frame rate $[\mathrm{fps}]$ & 10 \\
\hline
\end{tabular}

ferent motions. This means that the translational or rotational direction can also be identified because the azimuth component of optic flow becomes zero in the moving or rotational-axis direction. However, note that optic flow cannot be obtained in a hemisphere upper than horizontal level. Thus, the directions of image sensors along an elevation angle should be decided considering the field of view of image sensors and the expected attitude change.

From the comparison between Tables 3 and 4 and between Figs. 4 and 6, the estimation accuracy of $r$ is better than those for $p$ and $q$. This is because $r$ motion generates a distinctive flow pattern with constant magnitudes along azimuth directions. However, contrary in near horizontal regions, resemble flow patterns can be generated in a downward direction, and the estimation accuracy sometimes degrades considerably for the limited region of optic flow in a real image sensor. Consider Figs. 7 and 8; Fig. 7 shows an example of optic flow produced by a positive directional $u$ motion, while Fig. 8 is optic flow obtained by a negative directional $q$ rotation. When optic flow is obtained in a whole spherical image plane, the difference between Figs. 7 and 8 is obvious. However, when the region along the elevation angle is restricted in $\beta=150 \sim 180$ degrees, the optic flows in the both figures are quite similar. Thus, it is difficult to distinguish $u$ and $-q$ motion from optic flow obtained from the restricted image region. This similarity can be appeared in other variables, e.g. between $v$ and $p$ motion.

\section{Experiments with a Real System}

Figure 9 shows the optic flow sensor used in experiments. Table 5 shows the specifications of the sensor; optic flow can be obtained at points $(79 \times 59)$ in the field of view $(45.1 \mathrm{deg} \times 34.6 \mathrm{deg})$ at every 0.1 second.

Because of the estimation difficulty denoted of the last paragraph in the previous section, two optic flow sensors are used in

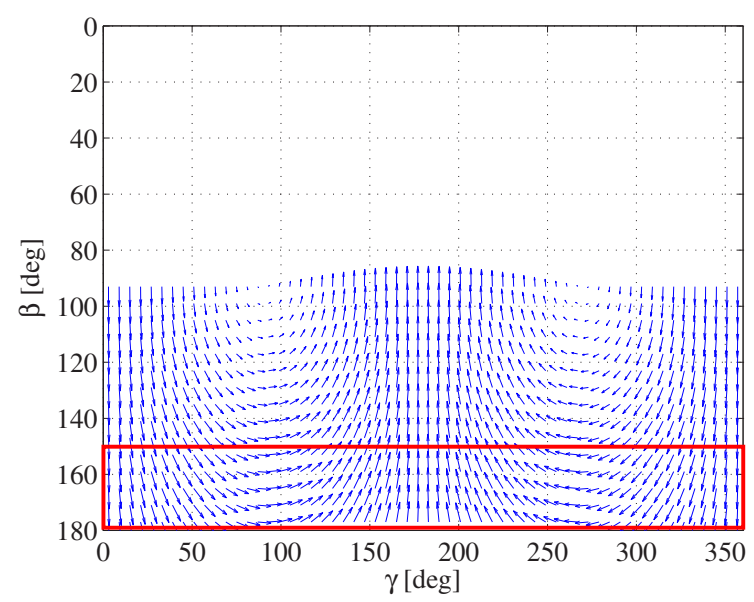

Fig. 8. Example of optic flow produced by $-q$ motion.

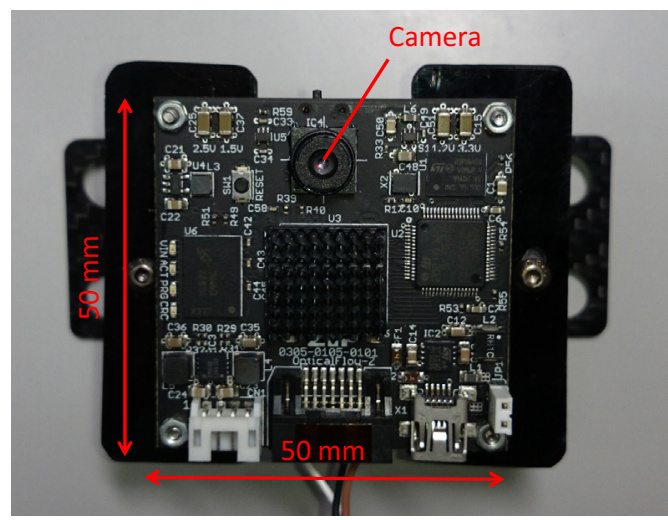

Fig. 9. Optic flow sensor.

the experiments. These optic flow sensors have been mounted on a multirotor vehicle which is a model of a space probe, and the estimation accuracy of the WFI of optic flow has been evaluated in the experiments. On the other hand, for the comparison of the estimation results, motion variables of the vehicle have been measured with a motion capture system.

Table 6 shows the directions of optical axes in three patterns used in the experiments. Subscripts of 1 and 2 in the table mean the index of two cameras. As can be seen, two azimuth angles are fixed through the experiments, and the effects of the elevation angles on estimation accuracy are examined.

As an example, Fig. 10 shows the time histories of the estimation results in Case 3b. Red lines are state parameters estimated from WFI of optic flow, while black lines indicate the motion variables measured by the motion capture system. The motion capture system can measure the position data of target markers with milimeter-accuracy in $50 \mathrm{~Hz}$. However, due to noises and discretization errors, the accuracy of the velocity evaluated from differentiating the position data becomes lowered. Thus from this figure, simply it can be found that the velocity estimation from WFI of optic flow with real sensors is

Table 6. Directions of optical axis in three patterns used in the experiments.

\begin{tabular}{|c|c|c|c|}
\hline \multicolumn{2}{|c|}{} & $\left(\beta_{1}, \beta_{2}\right)[\mathrm{deg}]$ & $\left(\gamma_{1}, \gamma_{2}\right)[\mathrm{deg}]$ \\
\hline \multirow{3}{*}{ Case 3 } & $\mathrm{a}$ & $(150,150)$ & $(0,90)$ \\
\cline { 2 - 4 } & $\mathrm{b}$ & $(135,135)$ & $(0,90)$ \\
\cline { 2 - 4 } & $\mathrm{c}$ & $(120,120)$ & $(0,90)$ \\
\hline
\end{tabular}



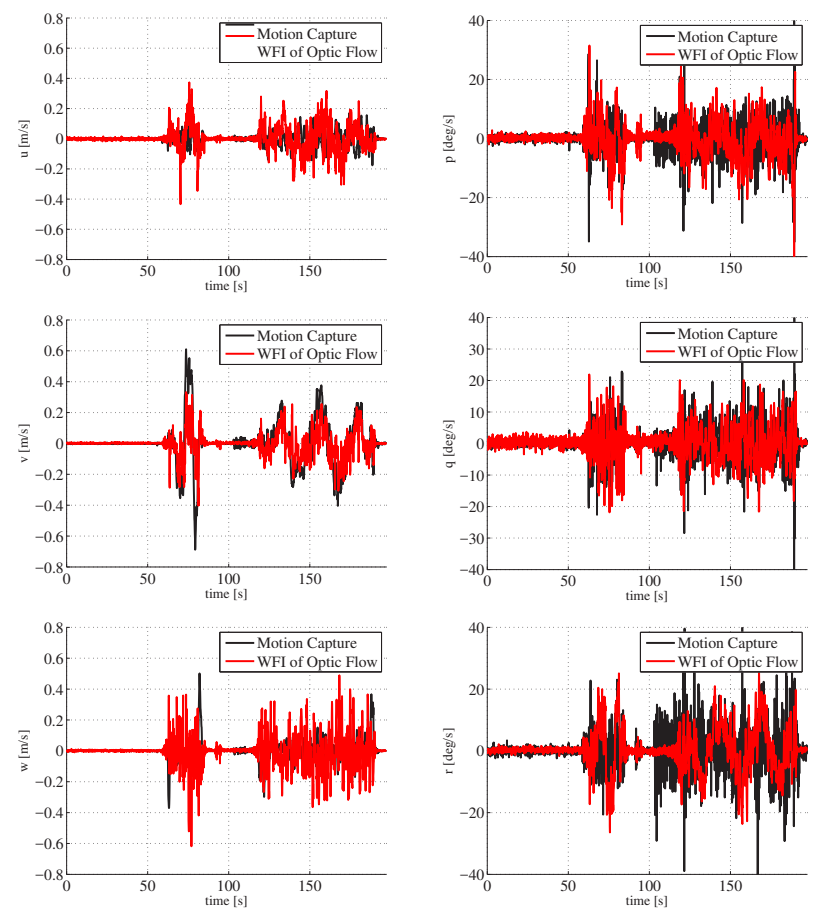

Fig. 10. Time histories of the estimation results in Case $3 b$.

Table 7. Mean values of estimation error in Case 3.
\begin{tabular}{|c|c|c|c|c|c|c|}
\hline & $\begin{array}{c}u \\
{[\mathrm{~m} / \mathrm{s}]}\end{array}$ & $\begin{array}{c}v \\
{[\mathrm{~m} / \mathrm{s}]}\end{array}$ & $\begin{array}{c}w \\
{[\mathrm{~m} / \mathrm{s}]}\end{array}$ & $\begin{array}{c}p \\
{[\mathrm{deg} / \mathrm{s}]}\end{array}$ & $\begin{array}{c}q \\
{[\mathrm{deg} / \mathrm{s}]}\end{array}$ & $\begin{array}{c}r \\
{[\mathrm{deg} / \mathrm{s}]}\end{array}$ \\
\hline Case 3a & 0.10 & 0.14 & 0.11 & 11.54 & 12.33 & 5.91 \\
\hline Case 3b & 0.05 & 0.06 & 0.07 & 4.92 & 4.71 & 5.55 \\
\hline Case 3c & 0.06 & 0.08 & 0.06 & 4.58 & 3.59 & 5.27 \\
\hline
\end{tabular}

in acceptable accuracy. Especially, the accuracy of $v$ parameter is reasonable, because the vehicle moves along $\pm y$ direction in the experiments; this is consistent to the discussion in the simulations. Table 7 and Fig. 11 show the mean values and the standard deviations for the estimation errors in three cases. From these results, it can be seen that the estimation accuracy becomes better when the optical axes of the image sensors approach to a horizontal direction; this is also consistent to the discussion in the simulations. Note that the standard deviations of velocities in Fig. 11 are much smaller than those in Fig. 4 or Fig. 6. This is mainly caused by two reasons: the translational velocities in the experiments were smaller than those in the simulations, and two optic flow sensors were used in the experiments while the simulations were calculated for one sensor. In the same reasons, the standard deviations of angular rate in Fig. 11 are larger than those in Fig. 4 or Fig. 6, because the angular rates in the experiments were larger than those in the simulations.
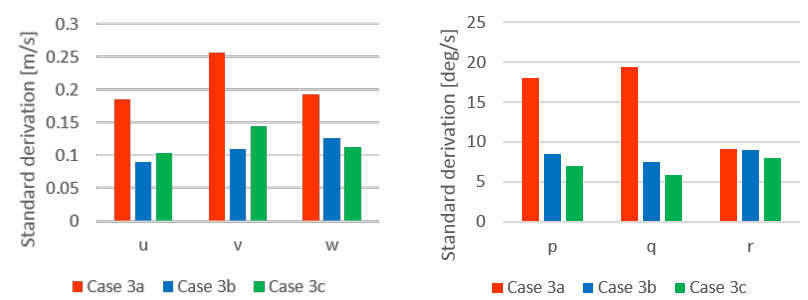

Fig. 11. Standard deviations of estimation error in Case 3.

\section{Conclusions}

State estimation through WFI of optic flow is expected as an autonomous guidance and navigation system because of its several attractive features. However, the region of an image sensor is limited in a real system, although the theory of WFI of optic flow assumes that optic flow is obtained in a whole spherical image surface. Thus, the effects of the directions of optical axes on the estimation accuracy were investigated in this paper. Numerical simulations examined the estimation accuracy of state parameters for the different azimuth and elevation directions of optical axes. The results indicate that under a limit field of view, one optic flow sensor frequently encounters difficulty to distinguish motion variables from WFI of optic flow. This means that multiple optic flow sensors are necessary to obtain reasonable estimation accuracy in real systems. Furthermore, the tendency between the directions of optic flow sensors and the estimation accuracy was verified in some experiments by using a multirotor vehicle with two optic flow sensors.

\section{References}

1) Thakoor, S., Morookian, J., Chahl, J., Hine, B. and Zornetzer, S.: BEES: Exploring Mars with Bioinspired Technologies, Computer, 37 (2004), pp. 38-47.

2) Izzo, D., Weiss, N. and Seidl, T.: Constant-Optic-Flow Lunar Landing: Optimality and Guidance, Journal of Guidance, Control, and Dynamics, 34 (2011), pp. 1383-1395.

3) Gibson, J.: The Perception of the Visual World, Houghton Mifflin, Oxford, England, U.K., (1950), pp. 117-144.

4) Koenderink, J. J. and van Doorn, A. J.: Facts on Optic Flow, Biological Cybernetics, 56 (1987), pp. 247-254.

5) Egelhaaf, M., Kern, R., Krapp, H. G., Kretzberg, J., Kurtz, R. and Warzecha, A.: Neural Encoding of Behaviourally Relevant VisualMotion Information in the Fly, Trends in Neurosciences, 25 (2002), pp. 96-102.

6) Humbert, J. S.: Bio-Inspired Visuomotor Convergence in Navigation and Flight Control Systems, Ph. D. Dissertation, Mechanical Engineering Department, California Inst. of Technology, Pasadena, CA (2005).

7) Conroy, J., Gremillion, G., Ranganathan, B. and Humbert, J. S.: Implementation of Wide-Field Integration of Optic Flow for Autonomous Quadrotor Navigation, Autonomous Robots, 27 (2009), pp. 189-198.

8) Humbert, J. S. and Hyslop, A. M.: Bio-Inspired Visuomotor Convergence, IEEE Transactions on Robotics, 26 (2010), pp. 121-130.

9) Hyslop, A. M., Krapp, H. G. and Humbert, J. S.: Control Theoretic Interpretation of Directional Motion Preferences in Optic Flow Processing Interneurons, Biological Cybernetics, 103 (2010), pp. 353-364.

10) Hyslop, A. M. and Humbert, J. S.: Autonomous Navigation in Three-Dimensional Urban Environments Using Wide-Field Integration of Optic Flow, Journal of Guidance, Control, and Dynamics, 33 (2010), pp. 147-159.

11) Shoemaker, M. A. and Hokamoto, S.: Application of Wide-Field Integration of Optic Flow to Proximity Operations and Landing for Space Exploration Missions, Advances in the Astronautical Sciences, 142 (2011), pp. 23-36.

12) Sakamoto, H. and Hokamoto, S.: Attitude Angle Estimation of Space Probe through Wide-Field Integration of Optic Flow, Trans. of JSASS Aerospace Technology Japan., 12, ists29 (2014), pp.Pd_41-Pd_46. 